\title{
EQUICONTINUITY OF ITERATES OF CIRCLE MAPS
}

\author{
ANTONIOS VALARISTOS \\ Department of Mathematics \\ University of Florida \\ Gainesville, FL 32611 USA \\ (antonis@math.ufl.edu)
}

(Received September 6, 1994)

\begin{abstract}
Let $f$ be a continuous map of the circle to itself. Necessary and sufficient conditions are given for the family of iterates $\left\{f^{n}\right\}_{n=1}^{\infty}$ to be equicontinuous.
\end{abstract}

KEY WORDS AND PHRASES. Equicontinuity, period of a periodic point 1992 AMS SUBJECT CLASSIFICATION CODES. 54H20.

\section{INTRODUCTION.}

Let $C^{0}(X, Y)$ denote the set of continuous maps from $X$ to $Y, I$ a closed unit interval and $S^{1}$ the circle. Let $f \in C^{0}(I, I)$ and suppose that the family of iterates of $f$, i.e. $\left\{f^{n}\right\}_{n=1}^{\infty}$, is equicontinuous. Let $F_{1}$ and $F_{2}$ denote the fixed point set of $f$ and $f^{2}$ respectively. A. M. Bruckner and T. Hu [4] have shown that $\left\{f^{n}\right\}$ is equicontinuous if and only if $F_{2}=\cap_{n=1}^{\infty} f^{n}(I)$. We show that for maps of the circle the following result holds:

THEOREM. Let $f \in C^{0}\left(S^{1}, S^{1}\right)$. Then $\left\{f^{n}\right\}_{n=1}^{\infty}$ is equicontinuous if and only if one of the following holds:

(1) $f$ is conjugate to a rotation.

(2) $F_{1}$ consists of exactly two distinct points and every other point on $S^{1}$ has period two.

(3) $F_{1}$ consists of single point and $F_{2}=\cap_{n=1}^{\infty} f^{n}\left(S^{1}\right)$.

(4) $F_{1}=\cap_{n=1}^{\infty} f^{n}\left(S^{1}\right)$.

2. PRELIMINARIES.

Let $f \in C^{0}\left(S^{1}, S^{1}\right)$. We think of the circle $S^{1}$ as $R / Z$ and for $x, y \in S^{1}$ with $x \neq y$ we denote by $[x, y]$ the closed interval from $x$ counterclockwise to $y$. Let $d(x, y)$ denote the $\min \{|[x, y]|,|[y, x]|\}$ where $|[x, y]|$ is the length of the interval $[x, y]$. For any nonnegative integer $n$ define $f^{n}$ inductively by $f^{n}=f \circ f^{n-1}$, where $f^{0}$ is the identity map on $S^{1}$. A point $x \in S^{1}$ is a periodic point of $f$ if there is a positive integer $n$ such that $f^{n}(x)=x$. The least such $n$ is called the period of $x$. A point of period one is called a fixed point. Let $F_{n}$ denote the fixed point set of $f^{n}, \forall n \geq 1$ and $P(f)$ the set of periodic points of $f$.

If $x \in S^{1}$ then the trajectory of $x$ is the sequence $\gamma(x, f)=\left\{f^{n}(x)\right\}_{n \geq 0}$ and the w-limit set of $x$, $\omega(x, f)=\cap_{m \geq 0} \overline{U_{n \geq m} f^{n}(x)}$. Equivalently, $y \in \omega(x, f)$ if and only if $y$ is a limit point of the trajectory $\gamma(x, f)$, i.e. $f^{n_{k}}(x) \rightarrow y$ for some sequence of integers $n_{k} \rightarrow \infty$. Let $\mathcal{F}=\left\{f, f^{2}, f^{3}, \ldots\right\}$. The family of functions $\mathcal{F}$ is said to be equicontinuous if given $\epsilon>0$ there exists a $\delta>0$ such that $d\left(f^{i}(x), f^{i}(y)\right)<\epsilon$ whenever $d(x, y)<\delta$ for all $x, y \in S^{1}$ and all $i \geq 1$.

The following theorem is proved by J. Cano [5]: 
THEOREM A. Let $f \in C^{0}(I, I)$ such that $\left\{f^{n}\right\}_{n=1}^{\infty}$ is equicontinuous. Then $F_{1}$ is connected and if it is non-degenerate then $F_{1}=P(f)$.

The next theorem which is given in [4] and is due to A. M. Bruckner and Thakyin Hu (only if) and W. Boyce (if):

THEOREM B. Let $f \in C^{0}(I, I)$. Then $\left\{f^{n}\right\}_{n=1}^{\infty}$ is equicontinuous if and only if $\cap_{n=1}^{\infty} f^{n}(I)=F_{2}$.

Combining these two theorems we get the following corollary:

COROLLARY. Let $f \in C^{0}(I, I)$. If $f$ has a periodic point of period $n>2$, then $\left\{f^{n}\right\}_{n=1}^{\infty}$ cannot be equicontinuous.

\section{RESULTS}

Let $f \in C^{0}\left(S^{1}, S^{1}\right)$ such that $\left\{f^{n}\right\}_{n=1}^{\infty}$ is equicontinuous. We consider three cases:

(I) $f$ has a fixed point on $S^{1}$.

(II) the smallest period of the periodic points of $f$ on $S^{1}$ is $n \geq 2$.

(III) $f$ has no periodic points on $S^{1}$.

We start with case (I). The basic result of this case is Theorem 1. We first show the following four lemmas:

LEMMA 1. Let $f \in C^{0}\left(S^{1}, S^{1}\right)$ such that $\left\{f^{n}\right\}_{n=1}^{\infty}$ is equicontinuous. Suppose that there is a fixed point $p$ on $S^{1}$, and let $J$ be the component of $F_{2}$ containing $p$. If $J$ is either $\{p\}$ or a proper closed interval containing $p$ then there exists an open interval $K$ containing $J$ such that $\omega(x, f) \subseteq J$, for every $x$ in $K$.

PROOF. First suppose that $J=\{p\}$. Let $\epsilon=\left|S^{1}\right| / 4>0$. By equicontinuity of $\left\{f^{n}\right\}_{n=1}^{\infty}$ there is an open interval $K$ containing $p$ such that for every $x$ in $K$ and for every $n \geq 1, d\left(f^{n}(x), p\right)<\epsilon$. Define $L=\overline{n_{j=0}^{\infty} f^{j}(K)}$. Then $L$ is a closed, proper, invariant interval. By previous results on the interval (Theorems A and B), the fixed point set of $f \mid L$ and $f^{2} \mid L$ is connected and therefore, it is $\{p\}$. Moreover, by the above corollary all periodic points of $\left.f\right|_{L}$ have period 1 or 2 . But the fixed point $p$ is the only periodic point of $f$ in $L$. Therefore $P(f)=F_{1}$ and by [2] the $\omega$-limit points coincide with the fixed points. Hence $p$ is the only $\omega$-limit point of $f$ in $L$ and thus $\omega(x, f)=\{p\}=J$, for every $x$ in $L$. Since $K \subset L, \omega(x, f)=\{p\}=J$, for every $x$ in $K$.

Now suppose that $J$ is a proper closed interval containing $p$. Let $q_{1}$ and $q_{2}$ be the endpoints of $J$, which are fixed points under $f^{2}$. Let $\epsilon=\left|S^{1}-J\right| / 4>0$. By equicontinuity of $\left\{f^{n}\right\}_{n=1}^{\infty}$, there is an open interval $K_{1}$ around $q_{1}$ such that for every $x$ in $K_{1}$ and for every $n \geq 1, d\left(f^{n}(x), f^{n}\left(q_{1}\right)\right)<\epsilon$. Similarly there is an open interval $K_{2}$ around $q_{2}$ such that for every $x$ in $K_{2}$ and for every $n \geq 1$, $d\left(f^{n}(x), f^{n}\left(q_{2}\right)\right)<\epsilon$. Define $L=\overline{\cup_{j=0}^{\infty} f^{j}\left(K_{1} \cup J \cup K_{2}\right)}$. Then $L$ is a closed, proper, invariant interval. By previous results on the interval (Theorems $A$ and B), the fixed point set of $f \mid L$ and $f^{2} \mid L$ is connected and therefore it is $J$. Moreover, by the Corollary all periodic points of $\left.f\right|_{L}$ have period 1 or 2 , which we know that lie in $J$. Since $P(f)$ is closed, by [2], it coincides with the set of $\omega$-limit points. Therefore $\omega(x, f) \subset J$, for every $x$ in $L$. Let $K=K_{1} \cup J \cup K_{2}$. Then $K \subset L$ and $\omega(x, f) \subset J$, for every $x$ in $K$.

LEMMA 2. Let $f \in C^{0}\left(S^{1}, S^{1}\right)$ such that $\left\{f^{n}\right\}_{n^{-1}}^{\infty}$ is equicontinuous. Suppose that there is a fixed point $p$ on $S^{1}$, and let $J$ be the component of $F_{2}$ containing $p$. Define $S=\left\{x \in S^{1}: \omega(x, f) \subseteq J\right\}$. Then $S=S^{1}$.

PROOF. There are three cases:

(i) $J=\{p\}$, (ii) $J$ is a proper closed interval containing $p$ and (iii) $J=S^{1}$.

If (iii) holds then obviously $S=J=S^{1}$.

Therefore assume (i) and (ii) hold. Then by Lemma 1, there exists an open interval $K$ containing $J$ such that $\omega(x, f) \subseteq J$, for every $x$ in $K$. Note that $S$ is nonempty since $S \supseteq K$. First we show that $S$ is 
open: Let $x \in S$. Then $\omega(x, f) \subseteq J$, by definition of $S$. Choose $N$ large enough such that $f^{N}(x) \in K$. By continuity of $f^{N}$ there is a neighborhood $U$ of $x$ such that if $y \in U$ then $f^{N}(y) \in K$. But then $\omega\left(f^{n}(y), f\right)=\omega(y, f) \subseteq J$ and $y \in S$. Therefore $S$ is open.

Let $T$ be the component of $S$ containing $J$ and therefore $K$, as well. Then $T$ is open and connected. We will show that $T=S^{1}$. Suppose $T \neq S^{1}$. Then $S^{1}-T$ is a closed interval or a point. Let $J=\left[q_{1}, q_{2}\right]$ where possibly $q_{1}=q_{2}=p$.

Suppose first that $S^{1}-T$ is a closed interval. Let $z_{1}$ and $z_{2}$ be the endpoints of this closed interval such that $\left[z_{2}, z_{1}\right] \cap J=\emptyset$. Let $\epsilon=\frac{1}{2} \min \left\{d\left(q_{1}, z_{1}\right), d\left(q_{2}, z_{2}\right)\right\}$. By equicontinuity of $\left\{f^{n}\right\}$ at $z_{1}$, there is an open interval $V_{1}$ around $z_{1}$ such that for every $x$ in $V_{1}$ and for every $n \geq 1, d\left(f^{n}(x), f^{n}\left(z_{1}\right)\right)<\epsilon$. Let $x \in T$ such that $d\left(x, z_{1}\right)<\epsilon$. Since $\omega(x, f) \subseteq J$ and the orbit of $z_{1}$ stays by definition out of $T$, there exists a positive integer $k$ such that $d\left(f^{k}(x), f^{k}\left(z_{1}\right)\right)>\epsilon$, which is a contradiction.

Now suppose that $S^{1}-T=\{z\}$. Let $\epsilon=\frac{1}{2} \min \left\{d\left(z, q_{1}\right), d\left(q_{2}, z\right)\right\}$. By equicontinuity of $\left\{f^{n}\right\}$ at $z$ there is an open interval $V$ around $z$ such that for every $x$ in $V$ and for every $n \geq 1$, $d\left(f^{n}(x), f^{n}(z)\right)<\epsilon$. Since $\omega(x, f) \subseteq J$ for every $x \in T$ and $f(z)=z$ is a fixed point of $f$, we get a contradiction.

Hence $T=S^{1}$. Thus $S=\left\{x \in S^{1}: \omega(x, f) \subset J\right\}=S^{1}$.

LEMMA 3. Let $f \in C^{0}\left(S^{1}, S^{1}\right)$. If $F_{2}=S^{1}$ then $F_{1}$ cannot consist of exactly one point.

PROOF. Suppose that there is an $f \in C^{0}\left(S^{1}, S^{1}\right)$ such that $F_{2}=S^{1}$ and $F_{1}=\{p\}$. Let $z$ be a point on $S^{1}-\{p\}$ of period two. Let $K$ be the closed interval with endpoints $z$ and $f(z)$ which contains $p$ and let $L$ be the closed interval with the same endpoints that does not contain $p$. Since $f$ is a homeomorphism, we have two cases:

(i) $f(K)=K$ and $f(L)=L$ or (ii) $f(K)=L$ and $f(L)=K$.

If (i) holds then, since $f(L)=L$, there would be another fixed point of $f$ in $L$, which is a contradiction since $F_{1} \subset K$

If (ii) holds then $f(K)=L$ implies that $p$ cannot be a fixed point which is again a contradiction.

LEMMA 4. Let $f \in C^{0}\left(S^{1}, S^{1}\right)$. If $F_{2}=S^{1}$ and $F_{1}$ consists of more than two distinct points then $f$ is the identity on $S^{1}$.

PROOF. Assume that $F_{1}$ consists of exactly $k>2$ distinct fixed points $p_{1}, p_{2}, \ldots, p_{k}$. Let $L_{i}=\left[p_{i}, p_{i+1}\right]$ for $i=1,2, \ldots, k-1$ and $L_{k}=\left[p_{k}, p_{1}\right]$ so that the interior of each $L_{i}$ does not contain any fixed points. Then we have two cases: (i) $f\left(L_{i}\right)=L_{i}$ and (ii) $f\left(L_{i}\right)=S^{1}-L_{i}$.

If (i) holds then pick $x$ in the interior of $L_{i}$. Note that $f(x)$ is a point in the interior of $L_{i}$ and denote by $M_{x}$ the closed interval with endpoints $x$ and $f(x)$ which is free of fixed points. If $f\left(M_{x}\right)=M_{x}$ then there would be another fixed point in $M_{x}$ contradicting that the interior of $L_{i}$ contains no fixed points. Thus the only choice is $x=f(x)$ for every $x \in L_{i}$ and $f \mid L_{i}$ is the identity map. The same argument applied to every $L_{i}$ shows that $f$ is the identity map on $S^{1}$.

If (ii) holds then there are points in $L_{i}$ which map onto the other fixed points contradicting that $F_{2}=S^{1}$.

THEOREM 1. Let $f \in C^{0}\left(S^{1}, S^{1}\right)$ such that $\left\{f^{n}\right\}_{n=1}^{\infty}$ is equicontinuous. Suppose that there is a fixed point $p$ on $S^{1}$. Then $f$ has periodic points of period at most two and $F_{2}$ is connected. Furthermore $F_{1}$ is either connected or it consists of exactly two distinct points and every other point on $S^{1}$ has period two. Moreover if $F_{1}$ is a nondegenerate interval then $F_{1}=P(f)$.

PROOF. Let $J$ be the component of $F_{2}$ containing $p$. There are three cases:

(i) $J=\{p\}$, (ii) $J$ is a proper closed interval containing $p$ and (iii) $J=S^{1}$.

Assume that (i) holds. Then, by Lemma $2, \omega(x, f)-\{p\}$ for every $x \in S^{1}$. Thus the fixed point $p$ is the only periodic point of $f$ on $S^{1}$ and hence $P(f)=F_{1}=F_{2}=\{p\}$ is connected.

Assume that (ii) holds. Then, by Lemma $2, \omega(x, f) \subseteq J$ for every $x \in S^{1}$ and the periodic points of $f$ on $S^{1}$ lie in $J$. By results on the interval applied to $f \mid J$, either $p$ is the unique fixed point of $f$ on $S^{1}$ or 
$F_{1}$ is a nondegenerate interval and the fixed points are the only periodic points of $f$ on $S^{1}$. In particular, both $F_{1}$ and $F_{2}$ are connected.

Assume that (iii) holds. Then all of the points of $S^{1}$ are periodic with period 1 or 2 and $F_{2}$ is connected. By Lemma 3, $F_{1}$ cannot consist of one point and by Lemma 4 if $F_{1}$ consists of more than two points then $f$ is the identity map. Otherwise $F_{1}$ consists of exactly two distinct points and every other point on $S^{1}$ has period two.

We now investigate case (II) where the smallest period of the periodic points of $f$ on $S^{1}$ is $n \geq 2$. The main result here is Theorem 2. We use Lemma 5 in the proof of the main theorem.

THEOREM 2. Let $f \in C^{0}\left(S^{1}, S^{1}\right)$ such that $\left\{f^{n}\right\}_{n=1}^{\infty}$ is equicontinuous. Suppose that the smallest period of the periodic points of $f$ on $S^{1}$ is $n \geq 2$. Then every point on $S^{1}$ is periodic with period $n$.

PROOF. Let $p$ be a periodic point of period $n$ on $S^{1}$. Then $f^{n}(p)=p$ and therefore $p$ is a fixed point of $f^{n}$. Applying Theorem 1 to $f^{n}$, we conclude that $F_{n}$ is either connected or it consists of exactly two distinct points and every other point on $S^{1}$ has period $2 n$.

We claim that there is no continuous map of the circle having two points of period two and every other point periodic of period four. Otherwise, if $g$ is such a map, let $p, g(p)$ be the two points of period two and let $K=[p, g(p)]$ and $L=[g(p), p]$. Since $g$ is a homeomorphism, we have two cases: (i) $g(K)=K$ and $g(L)=L$ or (ii) $g(K)=L$ and $g(L)=K$. In both cases $g^{2}(K)=K$. Hence if $x \in K$ is a point of period four then $g^{2}(x) \neq x$ and $g^{2}(x) \in K$. If $M$ is the closed interval with endpoints $x$ and $g^{2}(x)$ lying in $K$ then $g^{2}(M)=M$. Therefore $M$ contains a periodic point of period two, contradicting the assumption that $p$ and $g(p)$ are the only points of period two and every other point has period four.

Hence $F_{n}$ is connected. Suppose that $F_{n} \neq S^{1}$. Then $F_{n}$ is a proper closed interval containing the orbit of $p$ under $f$. Moreover $f\left(F_{n}\right) \subset F_{n}$. This implies that $f$ has a fixed point on $S^{1}$, contradicting the hypothesis that the smallest possible period of the periodic points is $n>1$. Hence $F_{n}=S^{1}$.

For a proof of the following see [7].

LEMMA 5. Let $f \in C^{0}\left(S^{1}, S^{1}\right)$. Suppose that there exists a positive integer $n \geq 2$ such that every point on $S^{1}$ is periodic with period $n$. Then $f$ is conjugate to a rational rotation.

Now we consider case (III) where $f \in C^{0}\left(S^{1}, S^{1}\right)$ has no periodic points and $\left\{f^{n}\right\}$ is equicontinuous. The main result here is listed in Theorem 3

Note that $f$ must be onto, since otherwise $f\left(S^{1}\right)=I$ is homeomorphic to a closed interval and $f(I) \subset I$, so $f$ has a fixed point. We shall adapt the techniques and use results due to J. Auslander and Y. Katznelson [1].

Let $x \in S^{1}$. In [1], $J_{x}$ is defined to be the largest interval containing $x$ such that $f^{m}(x) \notin J_{x}, \forall m \geq 1$. Denote by $z_{1}$ and $z_{2}$ the endpoints of $J_{x}$, where possibly $z_{1}=z_{2}=x$. The following are showed in [1]: $J_{x}$ is closed and $z_{1}, z_{2} \neq f^{k}(x)$ for $k \geq 1$. If $x, y \in S^{1}$ then $y \in \omega(x, f)$ if and only if $y$ is an endpoint of $J_{y}$. If $z_{1}$ and $z_{2}$ are the endpoints of $J_{x}$, then $f\left(z_{1}\right)$ and $f\left(z_{2}\right)$ are the endpoints of $f\left(J_{x}\right)$. Also $f\left(J_{x}\right) \cap J_{x}=\emptyset$ and $f^{m}\left(J_{x}\right)=J_{f^{m}(x)}, \forall m \geq 1$. The intervals $J_{f^{m}(x)}$ ( $m=0,1,2, \ldots)$ are pairwise disjoint and if $f(x)=f\left(x^{\prime}\right)$, then $J_{x}=J_{x^{\prime}}$. The sets $\left\{J_{x}\right\}$ form a partition of $S^{1}$ (that is, if $x, y \in S^{1}$ then $J_{x}=J_{y}$ or $J_{x} \cap J_{y}=\emptyset$ and $U_{x \in S^{1}} J_{x}=S^{1}$ ). Finally, at most countably many of the sets $J_{x}$ are non-degenerate $\left(J_{x} \neq\{x\}\right)$.

Before we show our result, we state the following theorem proved in [6] which concerns homeomorphisms.

THEOREM C. Let $f$ be an orientation preserving homeomorphism of $S^{1}$ to itself. For $x \in S^{1}$, let $R_{\alpha}(x)=x+\alpha(\bmod 1)$ denote irrational rotation by $\alpha$. Then $f$ is conjugate to some $R_{\alpha}$ if and only if some (all) orbits of $f$ are dense on $S^{1}$. 
We are now ready to show the following:

THEOREM 3. Let $f \in C^{0}\left(S^{1}, S^{1}\right)$ without periodic points and such that $\left\{f^{n}\right\}_{n=1}^{\infty}$ is equicontinuous. Then $f$ is conjugate to an irrational rotation $R_{\alpha}$.

PROOF. We first show that $\omega(x, f)=S^{1}$ for all $x \in S^{1}$. Since $y \in \omega(x, f)$ if and only if $y$ is an endpoint of $J_{y}$, it suffices to show that $\forall y \in S^{1}, J_{y}=\{y\}$. By the way of contradiction assume that $J_{y 0}$ is a non-degenerate interval. Since $f$ is onto, there exists $y_{1} \in S^{1}$ such that $f\left(J_{y 1}\right)=J_{y 0}$. Continuing in this way, we obtain a sequence of intervals $\left\{J_{y n}\right\}$ such that $f\left(J_{y n}\right)=J_{y n-1} \forall n \geq 1$. Since there are countably many non-degenerate such intervals on $S^{1}, \lim _{k \rightarrow \infty}\left|f^{-k}\left(J_{y 0}\right)\right|=0$. Hence $f^{k}$ maps arbitrarily small intervals onto $J_{y 0}$ (as $k \rightarrow \infty$ ) which contradicts equicontinuity. Therefore $\omega(x, f)=S^{1}$ for all $x \in S^{1}$.

This is equivalent to saying that all orbits of $f$ are dense in $S^{1}$. If $f(y)=f\left(y^{\prime}\right)$, then $J_{y}=J_{y^{\prime}}$ and hence $f$ is a homeomorphism. By Theorem $\mathrm{C}$ it follows immediately that $f$ is conjugate to an irrational rotation $R_{\alpha}$.

\section{PROOF OF THEOREM}

We first state the following three lemmas which can be shown to hold on any compact metric space.

LEMMA 6. Let $f, g \in C^{0}(X, X)$, where $X$ is a compact metric space. Suppose that $f$ is conjugate in $X$ to $g$ and that $\left\{g^{n}\right\}_{n=1}^{\infty}$ is equicontinuous. Then $\left\{f^{n}\right\}_{n=1}^{\infty}$ is equicontinuous.

LEMMA 7. Let $f \in C^{0}(X, X)$, where $X$ is a compact metric space. Let $k$ be a positive integer and $g=f^{k}$. Then $\left\{f^{n}\right\}_{n=1}^{\infty}$ is equicontinuous if and only if $\left\{g^{n}\right\}_{n=1}^{\infty}$ is equicontinuous.

LEMMA 8. Let $f \in C^{0}(X, X)$, where $X$ is a compact metric space. If $\left\{\left(\left.f\right|_{f(X)}\right)^{n}\right\}_{n=1}^{\infty}$ is equicontinuous then $\left\{f^{n}\right\}_{n=1}^{\infty}$ is equicontinuous.

Finally, we summarize the results to the following theorem.

THEOREM. Let $f \in C^{0}\left(S^{1}, S^{1}\right)$. Then $\left\{f^{n}\right\}_{n=1}^{\infty}$ is equicontinuous if and only if one of the following holds:

(1) $f$ is conjugate to a rotation.

(2) $F_{1}$ consists of exactly two distinct points and every other point on $S^{1}$ has period two.

(3) $F_{1}$ consists of a single point and $F_{2}=\cap_{n=1}^{\infty} f^{n}\left(S^{1}\right)$.

(4) $F_{1}=\cap_{n=1}^{\infty} f^{n}\left(S^{1}\right)$.

PROOF. We suppose that $\left\{f^{n}\right\}$ is equicontinuous. First assume that $F_{1}=\emptyset$. If $f$ has no periodic points on $S^{1}$, then by Theorem $3, f$ is conjugate to an irrational rotation, so that (1) holds.

If the smallest period of the periodic points of $f$ on $S^{1}$ is $n \geq 2$, then By Theorem 2, every point on $S^{1}$ is periodic with period $n$. It follows by Lemma 5 that $f$ is conjugate to a rational rotation, so that (1) holds again.

Now assume that $F_{1} \neq \emptyset$. Then by Theorem $1, f$ has periodic points of period at most two. If $F_{1}$ is not connected, then by Theorem 1 it consists of exactly two distinct points and every other point of $S^{1}$ has period two, so that (2) holds.

If $F_{1}$ is connected then it consists of (i) a single point, (ii) a proper interval or (iii) the whole circle.

(i) First assume that $F_{1}$ consists of a single point $p$. Note that by Theoreml, $F_{2}$ is a connected proper interval of $S^{1}$. Moreover by Lemma 2, we have that for every $x \in S^{1}, \omega(x, f) \subseteq F_{2}$. As in the proof of Lemma 1, there exists an open interval $K$ containing $F_{2}$ such that if $L=\overline{\cup_{j=0}^{\infty} f^{j}(K)}$ then $L$ is a proper interval. Of course $L$ is also closed and invariant. For $x \in S^{1}$, since $\omega(x, f) \subseteq F_{2} \subset K \subset L$, there exists a positive integer $N$ such that $f^{N}(x) \in K$. Then $f^{m}(x) \in L$ for every $m \geq N$. By continuity of $f^{N}$ there exists an open neighborhood $V_{x}$ of $x$ such that $f^{N}\left(V_{x}\right) \in K$ and hence $f^{m}\left(V_{x}\right) \in L$ for every $m \geq N$. Note that for each $x \in S^{1}$ the collection $\left\{V_{x}\right\}_{x \in S^{1}}$ forms an open cover of $S^{1}$. By compactness of $S^{1}$ there exists a finite subcover, which we denote by $\left\{V_{i}\right\}_{i=1, \ldots l}$. Consequently, for every $V_{i}$ there exists a positive integer $N_{i}$ such that $f^{N_{i}}\left(V_{i}\right) \subset K$, for $i=1,2, \ldots l$ 
and $f^{m_{2}}\left(V_{i}\right) \subset L$, for every $m_{i} \geq N_{i}$ and for $i=1,2, \ldots l$. Choose $N=\max \left\{N_{1}, \ldots, N_{l}\right\}$. Then $f^{m}\left(V_{i}\right) \subset L$, for every $m \geq N$ and $i=1,2, \ldots l$. Thus $f^{m}\left(S^{1}\right) \subset L$ for every $m \geq N$. By Theorem B, $\cap_{n=1}^{\infty} f^{n}(L)=F_{2}$. Since $f^{m}\left(S^{1}\right) \subset L$, for every $m \geq N$, it follows that $\cap_{n=1}^{\infty} f^{n}(L)=\cap_{n=1}^{\infty} f^{n}\left(S^{1}\right)=F_{2}$. Hence (3) holds.

(ii) Now assume that $F_{1}$ is a proper interval of $S^{1}$. We know by Theorem 1, that $F_{1}$ coincides with the set of periodic points of $f$. By an argument similar to the above applied to $F_{1}$, we can see that $F_{1}=\cap_{n=1}^{\infty} f^{n}\left(S^{1}\right)$ and hence (4) holds.

(iii) If $F_{1}=S^{1}$ then obviously (4) holds again.

This concludes one direction of the proof, namely that if $\left\{f^{n}\right\}_{n=1}^{\infty}$ is equicontinuous then one of (1), (2), (3) or (4) holds. Now we will show that all of these four cases imply that $\left\{f^{n}\right\}_{n=1}^{\infty}$ is equicontinuous.

Suppose that (1) holds i.e. $f$ is conjugate to a rotation $R$. Then $R^{n}$ is an isometry for every $n \geq 1$ and therefore $\left\{R^{n}\right\}$ is equicontinuous. It follows by Lemma 6 that $\left\{f^{n}\right\}$ is equicontinuous as well.

Suppose that (2) holds i.e. $F_{1}$ consists of exactly two distinct points and every other point on $S^{1}$ has period two. Then $f^{2}$ is the identity on $S^{1}$. Therefore $\left\{f^{2 n}\right\}$ is equicontinuous. It follows by Lemma 7 that $\left\{f^{n}\right\}$ is equicontinuous as well.

Suppose that (3) holds i.e. $F_{1}$ consists of a single point and $F_{2}=\cap_{n=1}^{\infty} f^{n}\left(S^{1}\right)$. Then $f\left(S^{1}\right) \neq S^{1}$, since otherwise $F_{2}=S^{1}$ and we have seen in Lemma 3 that there is no continuous map of the circle with one fixed point and every other point of period two. Hence $f\left(S^{1}\right)$ is a proper interval of $S^{1}$ and $\left.f\right|_{f\left(S^{1}\right)}: f\left(S^{1}\right) \rightarrow f\left(S^{1}\right)$ is a continuous map of the interval with fixed point set of $\left(\left.f\right|_{f\left(S^{1}\right)}\right)^{2}$ equal to $F_{2}$. Since $\cap_{n=1}^{\infty}\left(\left.f\right|_{f\left(S^{1}\right)}\right)^{n}\left(f\left(S^{1}\right)\right)=F_{2}$, it follows by Theorem B, that $\left\{\left(\left.f\right|_{f\left(S^{1}\right)}\right)^{n}\right\}_{n=1}^{\infty}$ is equicontinuous. By Lemma 8 we get that $\left\{f^{n}\right\}_{n=1}^{\infty}$ is equicontinuous.

Finally suppose that (4) holds i.e. $F_{1}=\cap_{n=1}^{\infty} f^{n}\left(S^{1}\right)$. If $S^{1}=f\left(S^{1}\right)$ then $F_{1}=S^{1}$ and the identity map is equicontinuous. If $f\left(S^{1}\right)$ is a proper interval of $S^{1}$ then $F_{1}$ is a point or a proper interval of $S^{1}$. It follows that $\left.f\right|_{f\left(S^{1}\right)}: f\left(S^{1}\right) \rightarrow f\left(S^{1}\right)$ is a continuous map of the interval such that its fixed point set equals the fixed point set of $f$ on $S^{1}$. Since $\cap_{n=1}^{\infty}\left(\left.f\right|_{f\left(S^{1}\right)}\right)^{n}\left(f\left(S^{1}\right)\right)=F_{1}$, it follows by Theorem B, that $\left\{\left(\left.f\right|_{f\left(S^{1}\right)}\right)^{n}\right\}_{n=1}^{\infty}$ is equicontinuous. By Lemma 8 we get that $\left\{f^{n}\right\}_{n=1}^{\infty}$ is equicontinuous.

ACKNOWLEDGMENT. The author wishes to thank Louis Block for his guidance and many helpful conversations.

\section{REFERENCES}

[1] AUSLANDER, J. and KATZNELSON, Y., Continuous maps of the circle without periodic points, Israel J. Math., 32 (1978), 375-381.

[2] BLOCK, L. S. and COPPEL, W. A., Dymamics in One Dimension, Lecture Notes in Mathematics, 1513, Springer-Verlag, Berlin, 1992.

[3] BOYCE, W., Г-compact maps on an interval and fixed points, Trans. Amer. Math. Soc., 160 (1971), 87-102.

[4] BRUCKNER, A. M. and HU, T., Equicontinuity of iterates of an interval map, Tamkang, J. Math., 21 (1990), 287-294.

[5] CANO, J., Common fixed points for a class of commuting mappings on an interval, Trans. Amer. Math. Soc., 86 (1982), 336-338.

[6] KATZNELSON, Y., Sigma-finite invariant measures for smooth mappings of the circle, $J$. D'Analyse Math., 31 (1977), 1-18.

[7] NITECKI, Z., Differentiable Dymamics, The M.I.T. Press, 1971. 


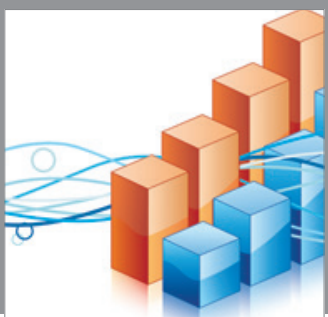

Advances in

Operations Research

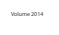

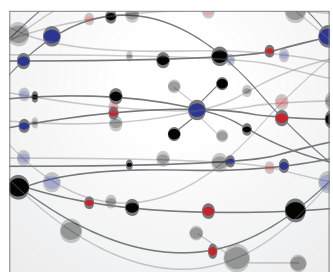

\section{The Scientific} World Journal
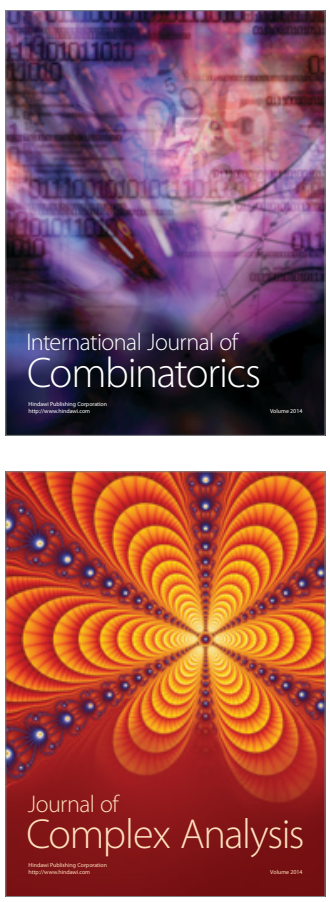

International Journal of

Mathematics and

Mathematical

Sciences
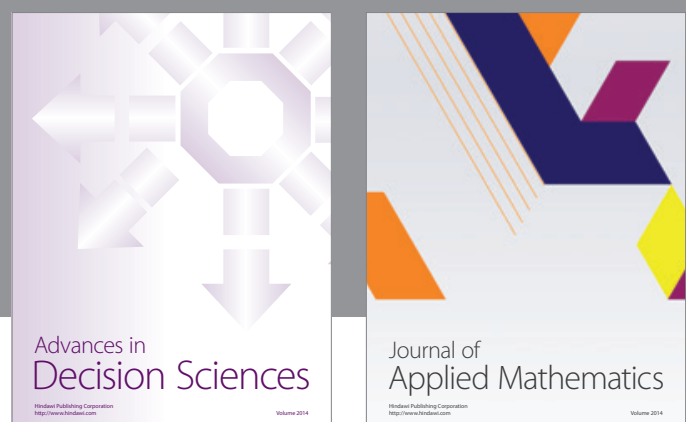

Journal of

Applied Mathematics
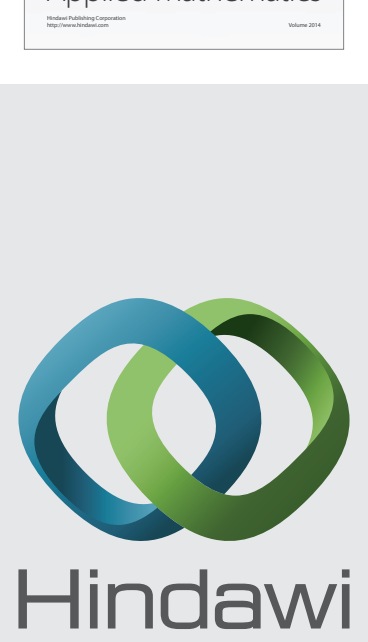

Submit your manuscripts at http://www.hindawi.com
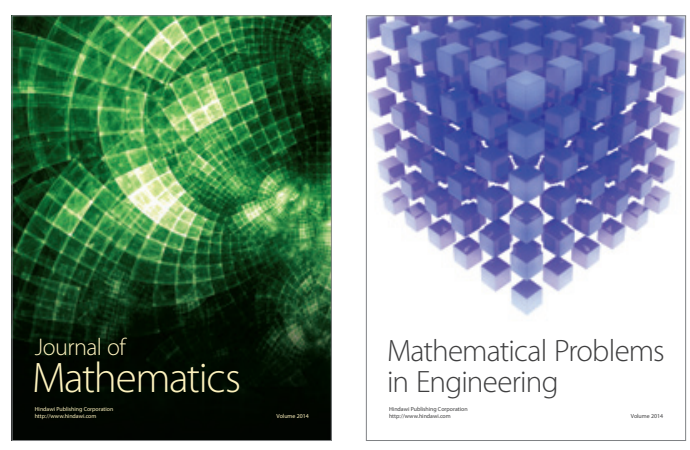

Mathematical Problems in Engineering
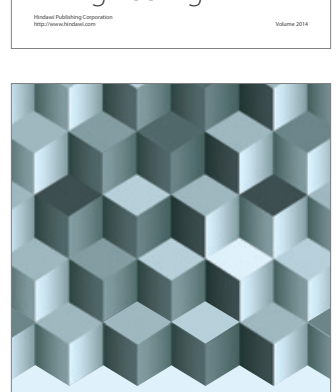

Journal of

Function Spaces
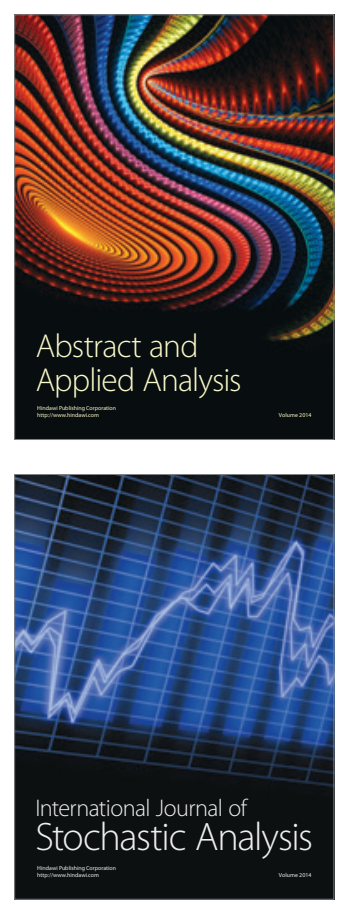

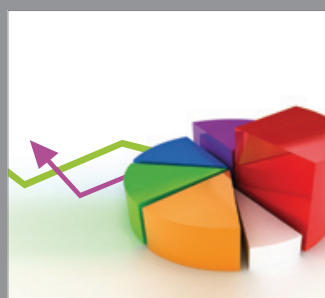

ournal of

Probability and Statistics

Promensencen
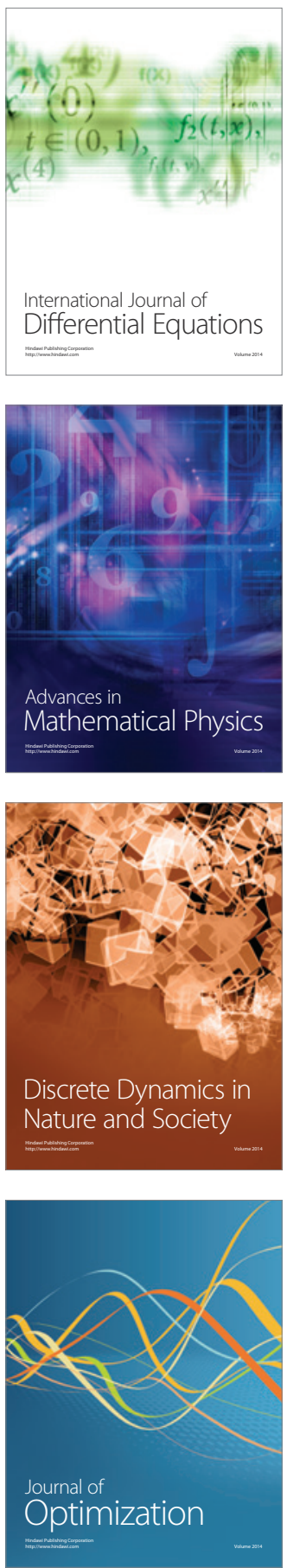